Cinémas

Revue d'études cinématographiques

Journal of Film Studies

\title{
Les paysages italiens. Entre cinéma et histoire
}

\section{Pierre Sorlin}

Volume 12, numéro 1, automne 2001

Le Paysage au cinéma

URI : https://id.erudit.org/iderudit/024866ar

DOI : https://doi.org/10.7202/024866ar

Aller au sommaire du numéro

Éditeur(s)

Cinémas

ISSN

1181-6945 (imprimé)

1705-6500 (numérique)

Découvrir la revue

Citer cet article

Sorlin, P. (2001). Les paysages italiens. Entre cinéma et histoire. Cinémas, 12(1),

35-48. https://doi.org/10.7202/024866ar

\section{Résumé de l'article}

Tandis que, dans la première moitié du XXe siècle, les représentations du paysage italien se réduisaient à quelques stéréotypes, un intérêt nouveau pour l'environnement se manifesta, autour de 1950, en littérature, dans les sciences humaines et au cinéma. Les cinéastes, cependant, ignorèrent les efforts des historiens qui tentaient de comprendre comment un territoire évolue avec ceux qui le peuplent; revenant, sans le savoir, aux leçons du positivisme, ils transformèrent les paysages en données permanentes propres à conditionner leurs habitants et, involontairement, fournirent de belles images aux agences de tourisme. 


\title{
Les paysages italiens. Entre cinéma et histoire
}

\section{Pierre Sorlin}

\begin{abstract}
RÉSUMÉ
Tandis que, dans la première moitié $d u x^{e}$ siècle, les représentations du paysage italien se réduisaient à quelques stéréotypes, un intérêt nouveau pour l'environnement se manifesta, autour de 1950, en littérature, dans les sciences humaines et au cinéma. Les cinéastes, cependant, ignorèrent les efforts des historiens qui tentaient de comprendre comment un territoire évolue avec ceux qui le peuplent; revenant, sans le savoir, aux leçons du positivisme, ils transformèrent les paysages en données permanentes propres à conditionner leurs habitants et, involontairement, fournirent de belles images aux agences de tourisme.
\end{abstract}

\begin{abstract}
Throughout the first half of the XXth century, representation of the Italian landscape consisted only of a number of stereotypes. But around 1950 a new interest in the environment arose in literature, the humanities, and cinema. Filmmakers, however, were unaware of the ways in which historians were attempting to understand how a place evolves alongside the people who inhabit it. Without realising it, they reverted to a positivist interpretation and transformed their landscapes into fixed givens with the power to establish their inhabitants'ways of life. In the process, they involuntarily provided travel agencies with postcard images of the landscape.
\end{abstract}

À l'aube du $\mathrm{Xx}^{\mathrm{e}}$ siècle, le cinéma italien et les paysages italiens n'existent pas. Implantés dans toute la péninsule, à Turin et à 
Rome, à Milan et à Naples, les studios s'engagent rapidement dans la production à faible coût de centaines de petits films qu'absorbent aussitôt le marché intérieur ou étranger. À l'instar des frères Lumière, certains producteurs envoient leurs opérateurs saisir sur le vif des événements exceptionnels ou observer de lointaines populations. Ces opérateurs travaillent vite, ne se concentrant que sur ce qui risque de gagner la faveur du public. Les "reportages" produits et distribués par Arturo Ambrosio l'un des premiers à assurer un service régulier d'actualités illustrent parfaitement cette façon de procéder: à Messine, où il enregistre les effets du tremblement de terre, le cameraman ne tente ni de présenter la ville ni de mesurer l'ampleur des dégâts; il se borne à quelques vues impressionnantes d'édifices détruits ou en train de s'effondrer.

A cette époque, les spectateurs restent fascinés avant tout par la reproduction du mouvement : ils aiment les scènes où personnages et animaux se déplacent; la variété des paysages italiens ne constitue pas leur préoccupation principale. $\grave{A}$ Naples, où un grand nombre de petites compagnies tournent en série mélodrames ou comédies, la baie et le port servent épisodiquement d'arrière-plan à une brève vue d'ensemble. Et lorsque les histoires ont pour protagonistes le peuple napolitain - artisans, vendeurs, pêcheurs - elles trouvent leur cadre naturel dans les rues étroites, sur les placettes ou dans les escaliers qui descendent à la mer. Le paysage intervient donc dans le cinéma italien, mais à titre de paysage unique ou typique. C'est celui de Sul lago di Como, de Malombra, de Rapsodia satanica: une combinaison de sommets alpins, de lacs et de torrents, d'arbres en fleur et de palmes ou, toujours selon le même rapport eau/montagne, une combinaison des collines liguriennes et des golfes méditerranéens. Un voilier quitte le rivage, se profile devant un soleil levant ou couchant, qui ménage d'harmonieux contre-jours, longe des rochers en surplomb, les vagues soulevées au passage de la barque ondulent mollernent, des oiseaux traversent le ciel : une vaste carte postale se déroule sous les yeux du spectateur.

Cette fixation sur un paysage a deux origines: littéraire et publicitaire. Gian Piero Brunetta a depuis longtemps mis en évidence les sources littéraires du premier cinéma italien (Brunetta, 
1980). Cependant, ce ne sont pas les adaptations de Dante, Manzoni ou Shakespeare qui font l'originalité des cas qui nous occupent, car toutes les cinématographies naissantes ont eu recours à ces ouvrages vulgarisés par de multiples éditions à bon marché. Ce qui importe ici, c'est la reconduction au cinéma de lieux communs véhiculés par la poésie, le roman et la chanson. Deux exemples suffiront pour montrer à quel point la thématique du souffle marin et de l'air purifiant des hauteurs s'est banalisée à la fin du XIX siècle. D'Annunzio: "Andrea retrouvait... ces effets inexprimables que produisent sur une âme vierge les lumières, les ombres, les couleurs, les odeurs de l'eau. La mer lui était une onde de paix à laquelle s'abreuvaient ses pensées " ( $\mathrm{Il}$ Piacere, 1889). Carducci: "Entends: le vent alpin salue de son frais murmure/la voile et lui dit: "Voile immaculée, vas" " (Rime e ritmi, 1899). Il n'est pas étonnant que des images aussi fortement baralisées par les feuilletons comme par la littérature intellectuellement légitime aient été reprises au cinéma.

Le paysage, cependant, est bien moins italien que suisse, ou plutôt alpin; c'est celui que vantent les affiches et les revues touristiques de l'époque. Partis la veille, en train, de Londres ou de Berlin, les voyageurs découvrent au matin eau, soleil et montagne lorsqu'ils débouchent sur le Lago Maggiore. Les lacs italiens, la Riviera, sont les lieux de villégiature favoris des gens fortunés et des diplomates qui choisissent bientôt Rapallo, Stresa, Locarno pour régler les affaires de l'Europe. En reprenant des images publicitaires, les cinéastes, inconsciemment sans doute, jouent un double jeu: ils rappellent aux classes dirigeantes des stations estivales synonymes, pour elles, de vacances et de plaisir; ils ouvrent aux travailleurs une fenêtre vers des sites qu'ils n'ont aucune chance de voir. Le cinéma italien n'a jamais complètement renoncé à sa veine subalpine qu'on suit jusqu'aux dernières décennies du $\mathrm{XX}^{\mathrm{e}}$ siècle dans des réalisations sentimentales et dramatiques comme Cuori solitari, La Stanza del vescovo, où sommets abrupts et plans d'eau, vaguement esquissés, suffisent pour évoquer une atmosphère superficiellement trouble et romantique.

Cette longue permanence met bien en relief le caractère ambigu de ce qu'on nomme "paysage". Qu'il soit littéraire, 
pictural ou cinématographique, le paysage correspond à une certaine conception de la nature: il est une convention partagée dans un milieu donné; il se réduit souvent à un petit nombre d'indices que le public sait immédiatement déchiffrer. Et pourtant, en un autre sens, le paysage n'est pas une notion purement culturelle: il s'agit des caractères particuliers d'un terroir tels qu'ils résultent de sa conformation physique, de son sol et de son climat, mais aussi, davantage peut-être, de la présence humaine, de la couverture végétale, des voies d'échange, de l'habitat qui s'y sont développés.

Lidée que chaque région possède une physionomie particulière, que le paysage n'est pas seulement "un état d'âme ", qu'il manifeste la vie d'une contrée, est d'origine récente (Ritter, 1963). Bien qu'ils aient traversé une grande partie de la péninsule, Goethe puis Stendhal n'ont guère parlé du pays lui-même, qu'ils ont moins observé que les monuments ou les villes et leurs habitants. La tardive "invention" du paysage a été justement mise en relation avec le développement des moyens de transport individuels - bicyclette, automobile - et avec l'essor d'un tourisme de masse auquel ne suffisaient plus les lacs ou la Riviera (Pugh, 1988). À ces influences évidentes, il faut ajouter celle du cinéma. $\mathrm{Si}$, au début du siècle, les principaux producteurs européens, Danois, Français et Italiens, ne se sont guère occupés du décor naturel, les Américains l'ont en revanche très vite mis à contribution. Durant sa période Biograph, Griffith explore l'Océan, la Prairie, les Rocheuses en vastes plans d'ensemble, qui ne se réduisent pas à une toile de fond, mais se trouvent pleinement intégrés à l'action. Au lendemain de la Première Guerre mondiale, Way Down East, The Covered Wagon, Kentucky Pride, The Wind, Sunrise révèlent aux Européens une manière pour eux inhabituelle de définir les personnages en fonction de leur milieu et de faire jouer à la nature le rôle de véritable protagoniste.

Pourtant, sur ce plan, Hollywood n'influença directement aucun cinéma. Le combat de l'individu avec les éléments, la valorisation épique des grands espaces restèrent des spécialités américaines. Mais, dans le cas particulier de l'Italie, et par d'autres voies, le sol, le climat, l'occupation humaine devinrent éga- 
lement des sujets cinématographiques. En prenant le contrôle de l'Istituto Luce - centre de production de documentaires et d'actualités - et en créant l'Istituto del cinema educatore, le fascisme se dote explicitement de deux instruments de propagande. Il s'agit pour lui, entre autres projets, de river les agriculteurs à la terre en les persuadant que les bonifications et la modernisation amélioreront leur existence, tout en fournissant ses bases à l'autarcie économique. Cet objectif initial se double par la suite d'un autre objectif: en faisant connaître la diversité de l'Italie, le cinéma attirera les voyageurs étrangers et, dans le cadre d'un loisir populaire à promouvoir, incitera les Italiens à visiter leur pays. Le caractère artificiel des documentaires qui mettent en scène une Italie imaginaire, exempte de problèmes, lancée à fond sur la voie du progrès, n'a pas besoin d'être à nouveau souligné (Brunetta, 1972). Notons surtout qu'en respectant les consignes du pouvoir, l'Istituto Luce se voit doter d'un excellent matériel et peut travailler dans des conditions particulièrement favorables. C'est là, et au Centro Sperimentale fondé un peu plus tard (1936), qu'une nouvelle génération de réalisateurs, de scénaristes et d'opérateurs, étudiant les films américains et se déplaçant en tournage à travers la péninsule, se prend d'intérêt pour l'environnement.

Au tournant du siècle, après la reconstruction, quand s'annonce le "miracle économique", les Italiens commencent à porter un autre regard sur le cadre naturel qui les entoure. Le cinéma joue alors un rôle modeste dans ce mouvement de curiosité : les villes restent le cadre privilégié d'une grande majorité de films; villages et campagnes demeurent souvent le vague arrièreplan d'une intrigue sans véritable ancrage géographique. Le tourisme et la navigation de plaisance, la multiplication des escales aériennes, l'essor de la mode italienne, les échanges de capitaux et de travailleurs, l'incessant va-et-vient des équipes de football et de leurs supporters contribuent bien davantage que le cinéma à provoquer, en Italie comme à l'étranger, le désir de visiter la péninsule. Ces différents facteurs ont été depuis longtemps décrits (Scimone, 1964), mais les études consacrées à l'économie n'ont pas pu mettre en évidence ce que Calvino appelle, en 1958, "une restitution de l'homme à la nature dans l'art et la 
littérature des dernières années » (Calvino, 1980, p. 37). Le succès imprévisible de Cristo si è fermato a Eboli, publié par Carlo Levi en 1945, avait montré, comme le dit encore Calvino, que "la nature n'était plus ressentie comme altérité " mais que, au contraire, toute distance tendait à s'effacer «entre l'homme et l'ensemble de la matière vivante". "La seule suggestion qui me touche et me secoue est la nature, le regard fixé sur la colline", écrivait Pavese peu avant de mourir (Pavese, 1952, p. 331), tandis que le poète Salvatore Quasimodo notait, à peu près en même temps, que chaque écrivain semblait maintenant se concentrer sur "un monde réduit à un paysage - sa propre terre" (Quasimodo, 1971, p. 128).

Évoquant ce qu'il observe autour de lui, Calvino n'hésite pas à parler d'un "bouleversement profond dans la géographie du monde culturel $\%$. L'affirmation, à première vue excessive, se comprend mieux si l'on se remémore la situation antérieure. L'idée dominante, sous le fascisme, était celle de patrie, de terre italienne. Le régime poussait de toutes ses forces à l'exaltation d'une péninsule unie et unanime, et il n'avait pas à fournir un grand effort pour convaincre des intellectuels à qui cette vision simple convenait parfaitement. Mais s'il y a eu au temps de Mussolini, comme le note Gian Piero Brunetta, un «mythe de la terre" (Brunetta, 1993, p. 122-128), il n'a guère été représenté au cinéma, en dehors des nombreux documentaires "Luce", que par certaines œuvres dirigées par Blasetti. L'action de Terra madre (1931), par exemple, se déroule dans une campagne quasi anonyme où les personnages sont les porte-parole d'une illusoire alliance des propriétaires et des travailleurs contre l'invasion par l'argent et le trafic routier. À côté de cette pesante apologie de la paix sociale, le film le plus représentatif, dans l'optique du pays, est 1860 (1934). Par ce film, Blasetti inaugure la tradition des itinéraires à travers toute la péninsule, tradition qui s'est prolongée jusqu'à la fin du siècle avec Ladro di bambini, Invito al viaggio, Domani accadrà, Al centro dell'area di rigore. Le protagoniste de 1860 , un berger, part de Sicile et remonte jusqu'à Gênes pour convaincre Garibaldi de venir libérer son île. Le seul paysage qui soit longuement filmé est celui de la Sicile mais, grâce à un artifice parfaitement maîtrisé, cette 
terre osseuse, blanche de calcaire et de poussière, captive, menacée, est présentée du point de vue de «l'occupant», autrement dit des mercenaires suisses payés par les Bourbon. Pendant le voyage, puis après le retour dans l'île, le pays, entendu comme sol et comme nature, disparaît au profit de ses habitants, tous différents et pourtant tous indéfectiblement italiens. Le Blasetti de 1860 se trouve en parfaite communion avec l'historiographie dominante, celle du livre de Gioacchino Volpe, LItalia in camino (1929), qui résume la manière dont on doit comprendre l'évolution récente du pays: rassemblées en une seule patrie, les régions vont dépasser ce qui, longtemps, marqua leur originalité.

Le renversement de perspective qui s'opère au milieu du siècle devient tout à. fait sensible si l'on met en parallèle Paisà et Viaggio in Italia, deux "itinéraires" dirigés par Rossellini à sept années seulement d'intervalle. Bien que Paisà soit une traversée de la péninsule du Sud au Nord, de la Sicile au delta du Pô, la nature n'y apparaît pas avant le dernier épisode. Le film montre une population blessée, bouleversée par le combat opposant sur son territoire des troupes étrangères, parvenant néanmoins, à travers une lutte commune, à fraterniser avec les libérateurs. Ce sont les Italiens, ici encore divers mais confrontés à la même épreuve, qui rissent le lien unissant les étapes du parcours. Les protagonistes de Viaggio in Italia, un couple d'Anglais, ne sillonnent que la Campanie, mais leur rapport au territoire est particulièrement original: d'abord insensibles, méfiants à l'égard de l'exploitation touristique, ils sont peu à peu saisis par l'atmosphère locale, par les traces du passé, par l'ardeur religieuse de la population. $\mathrm{Si}$, au sens strict, le paysage intervient relativement peu, l'ensemble de la région - terre, hommes et traditions devient un véritable acteur du film. La nouveauté consiste à la fois dans l'évocation d'une épaisseur temporelle, d'un passé encore vivant à l'époque où l'action se déroule (c'est-à-dire l'époque même du tournage), et dans la force attractive d'un territoire capable de marquer durablement ceux qui le traversent: en apprenant à voir ce qu'ils refusaient auparavant de regarder, en se laissant prendre par l'atmosphère du lieu, les époux au bord de la rupture surmontent leurs problèmes et parviennent à se rejoindre. 
La percée de l'histoire dans le présent et le magnétisme d'une terre vivante conferent à Viaggio in Italia son caractère exceptionnel. D'autres films, dans la seconde moitié du $\mathrm{XX}^{\mathrm{e}}$ siècle, font du pays davantage qu'une toile de fond et lui ménagent parfois une véritable fonction narrative - mais la dimension temporelle, l'ancrage du lieu dans une évolution à long terme, dans une histoire, leur échappe généralement. Au demeurant, Calvino, dressant en 1958 un vaste panorama de la vie intellectuelle, emploie le mot "histoire" comme un équivalent d'évolution politique et, tout en examinant différents secteurs de la production littéraire, ne dit pas un mot des études historiques. Pourtant, l'une des tendances marquantes de l'époque, en ce domaine, est le rapide essor des recherches sur le paysage. En Grande-Bretagne et en France, des historiens s'intéressent déjà depuis longtemps aux milieux dans lesquels les hommes ont vécu et vivent encore. Quant aux travaux italiens, ils ne furent pas une simple imitation des modèles européens antérieurs. Leur initiateur, Emilio Sereni, militant communiste et érudit, entendait sortir le marxisme de son engourdissement dans les conflits de classe en repartant d'une autre intuition de Marx, celle qui regardait la lutte des formations sociales contre les forces naturelles et contre d'autres formations sociales. Relayé par ceux de Biasutti (1962), de Lucio Gambi et de Carlo Ginzburg (1979), l'ouvrage pionnier de Sereni, Storia del paesaggio agrario italiano (1960), a révolutionné l'approche traditionnelle des terroirs italiens en substituant à la notion imprécise de région celle, souple et évolutive, de paysage.

L'idée de région croise un découpage administratif séculaire et des particularités locales dont, souvent, on a pris conscience au moment où elles étaient sur le point de changer. Ainsi, le Mezzogiorno (et le problème qu'il représente) est-il devenu une entité particulière quand des intellectuels, Fortunato, Salvemini, Nitti, frappés par une émigration de masse qui allait bouleverser l'économie agraire, ont dénoncé «la malédiction endémique qui s'exerce sur l'Italie méridionale». Le paysage, tel que le conçoivent les historiens, n'est pas une suite de clichés. Loin d'être fixe (perenne, disait Giustino Fortunato en parlant du Sud), il évolue d'une période à une autre, sorte de réseau relationnel variable 
qui prend une allure différente selon qu'on le considère sous l'angle des échanges économiques, sous celui des rapports culturels ou sous celui de l'habitat.

Aux paysages dynamiques des historiens se juxtaposent (s'opposent?) ceux des films. La coïncidence de deux images banales réalisées à moindres frais sous le fascisme n’a pas résisté à l'enrichissement des perspectives chez les cinéastes comme chez les chercheurs en sciences humaines. Cependant, dans la seconde moitié du XX⿳亠丷厂 siècle, l'idée de paysage s'est trouvée écartelée entre deux visions difficilement conciliables: le cinéma, tout en prenant conscience de la diversité du sol italien, a conforté la représentation préexistante des régions. Si l'on ne retient que des œuvres dans lesquelles le milieu naturel est traité autrement qu'en simple décor, et si l'on néglige la permanence du folklore subalpin, quatre « régions» filmiques se distinguent nettement: la pianura padana, immense plaine construite par les alluvions du Pô, la Toscane, l'Italie centrale, Sabine, Ciociaria, les Abruzzes, le Sud enfin, entité imprécise où se détache particulièrement la Sicile.

On a déjà signalé que le seul épisode de Paisà dans lequel le territoire ait une importance, le dernier, a pour cadre le delta du Pô. L'eau, la terre spongieuse, les marais, le brouillard humide semblent avoir fasciné une génération entière au milieu du siècle. Ainsi, le documentaire de Fernando Cerchio sur Comacchio et celui d'Antonioni sur les gens du Pô sont à peu près contemporains de Paisà. Viendront ensuite d'autres films sur les pêcheurs, sur les rizières, sur les canaux. Un programme de la BBC 2 consacré à Antonioni (Wall, 1997) met en évidence la logique qui mène de Gente del Po (tourné en 1943, monté en 1947) aux nombreuses réalisations ancrées dans les boues de la Padana. Ce documentaire d'Antonioni saisit les gens, pêcheurs ou habitants des rives, au fil du courant : le lent écoulement du fleuve semble user la terre et les hommes, effacer les uns et les autres dans la médiocrité sans horizon de la plaine. Suintante, gluante, polluée par les routes et les raffineries chez Antonioni (Il Grido, Deserto rosso), morne, interminable, sans issue chez Bertolucci (Prima della rivoluzione, Strategia del ragno, La Luna), la Padana engloutit tous ceux qui passent, les écrase sous son ciel bas, les désespère par ses interminables rangées de peupliers. 
Curieusement, sur un territoire où l'on ne fait pas vingt kilomètres sans rencontrer une agglomération, elle propose une figure de ce que serait l'infini.

La Toscane cinématographique est un monde de contradictions. Visuellement, elle prolonge une longue tradition picturale et, dans les films comme sur les tableaux, étale des collines aux pentes légères, hachurées de cyprès, où alternent prairies et champs de blé, des vignes plantées en treilles, des palazzi aux toits étagés, de larges chemins débouchant, sans crier gare, sur une allée d'arbres et au fond sur une église. En apparence un lieu ouvert, idéal pour le repos et la promenade. En fait, une zone souvent hostile que protègent son littoral peu accueillant, ses larges étendues forestières où la marche, parfois, se révèle difficile, ses routes caillouteuses, interminables, qui semblent protéger d'inaccessibles villages. Sur un fond de campagne tranquille et ensoleillée, la Toscane de Comencini (La Ragazza di Bube), de Malaparte (Cristo proibito), des Taviani (Il Prato, La Notte di San Lorenzo), de Luchetti (La Settimana della sfinge) est une terre mystérieuse et sombre où les sentiers sont trompeurs, où l'on tourne en rond et où l'on s'égare; c'est une contrée menaçante, pleine de cachettes, de grottes, de recoins, accueillante pour les touristes dans la seule mesure où ils lui restent étrangers.

Les zones rocheuses de l'Italie centrale sont, au cinéma, l'inverse des Alpes: aux vallées verdoyantes, aux rivières et aux lacs, elles opposent la blancheur d'un calcaire aride et poussiéreux, des crêtes rocailleuses, glissantes, des arbres rares, des fonds de torrents à sec. Ciociaria et les Abruzzes sont les terres le moins souvent représentées mais, par réaction peut-être contre l'indifférence dont on les entoure, Germi (Il Brigante di Tacca del Lupo) et De Santis (Non čè pace tra gli ulivi, Giorni d'amore, Uomini e lupi) les traitent avec une attention particulière. Leurs personnages arpentent des versants où le caillou glisse sous le pied, et ils cherchent une ombre, une eau peut-être qu'ils ne découvriront qu'au terme d'un long parcours. La montagne filmée se refuse au voyageur -- à tel point qu'elle disparaît des écrans après les années cinquante.

Région aux limites imprécises, appellation générique sans caractères géographiques définis, le Mezzogiorno ne laisse indifférent 
aucun cinéaste. Ce qui attire les regards, c'est davantage la société avec ses lourdeurs, ses résistances et ses drames que le pays luimême: émigration, industrialisation ratée, urbanisation frénétique, mafia et trafics en tous genres remplissent les films aux dépens de l'environnement naturel. Le Sud est tellement hors temps qu'il donne à voir une histoire immobile, un passé pétrifié sous le soleil où, du XVI siècle de Gabriella Rosaleva (La Sposa di San Paolo) au XIX siècle de Visconti (Il Gattopardo) et de Vancini (I fatti di Bronte), les lieux ont à peine changé. Col cuore fermo Sicilia, le documentaire de Gianfranco Mingozzi, résume cette étrangeté, entre sécheresse et luxuriance, entre des côtes accueillantes et un intérieur aride. Blafardes, poudreuses sous un soleil vertical, les zones montagneuses ont, chez De Seta (Banditi a Orgosolo) ou Rosi (Salvatore Giuliano), le caractère implacable que d'autres films donnent aux Abruzzes, mais la caméra les effleure et, mobile, inquiète, elle cherche d'autres horizons. Au bord de la mer, elle s'attarde au contraire chez Visconti (La Terra trema) sur la splendeur des soleils levants; elle flâne, chez Rossellini (Stromboli) ou Antonioni (L'Avventura), autour des îles, observant la vie des pêcheurs. Le Mezzogiorno cinématographique est indéfinissable, séduisant et fuyant, il affiche trop de caractères différents pour être réduit à un modèle simple comme l'est celui de la Padana ou même à une forme ambiguë comme celle de la Toscane.

Nous n'avons cité que quelques films parmi les mieux connus, mais une liste complète ne serait pas longue; le fait de donner une place importante aux paysages ne concerne, redisons-le, qu'un petit groupe de réalisations. L'une des raisons en est sans doute que ce regard curieux et sans concession s'accorde mal avec les images diffusées par les agences de tourisme. Le cinéma italien fréquente peu les plages qui attirent les visiteurs et, s'il le fait, c'est pour rire des foules agglutinées sur quelques mètres de sable. Quand on s'interroge sur la portée critique des films, on oublie souvent l'importance d'un décalage par rapport à l'idéologie dominante: le seul fait de problématiser la vision de la péninsule a représenté une sorte de démenti face aux illusions et à l'euphorie qu'entretenait la légende du «miracle».

Pourtant, tout en contredisant les slogans publicitaires, le cinéma s'est maintenu à bonne distance des recherches 
historiques sur le paysage: là où les cinéastes découvraient une personnalité géographique qu'ils s'efforçaient de traduire visuellement, les historiens traquaient des indices de variabilité. Ainsi, l'image cinématographique d'une Padana reconnaissable à sa platitude, à ses champs réguliers, à ses rangées de peupliers leur estelle apparue comme une simplification poétique alors qu'euxmêmes s'attachaient à souligner les contrastes, opposant les zones drainées - où de grosses exploitations spécialisées dans l'élevage intensif ont créé une campagne ouverte - soit aux régions de polyculture où mûriers et fruitiers délimitent des parcelles dont les couleurs changent avec l'assolement, soit aux aires plus sèches où la transhumance a longtemps empêché le quadrillage du sol. Contre l'individualisation d'une Toscane ou d'un Sud, les historiens ont fait valoir l'étonnante diversité physique de la façade occidentale de la péninsule, avec des mois de sécheresse aussi longs en Ligurie qu'en Sicile et une côte le plus souvent inhospitalière; ils ont tenté de montrer comment l'interférence de plusieurs époques de conquête puis d'abandon a modelé le territoire; ils ont souligné les effets de la colonisation grecque sur l'agriculture sicilienne, les effets de l'abandon de l'ager publicus aux sénateurs romains sur l'extension des latifundia en Toscane méridionale et les effets de la division des terres nobles en Campanie du Sud. Inutile de s'étendre davantage sur ces analyses minutieuses, notre objectif n'est ni de critiquer le cinéma au nom de l'érudition ni de rappeler, ce dont tout le monde est conscient aujourd'hui, qu'un film est une création et non une fenêtre ouverte sur la vie. La seule question pertinente consiste à se demander ce que le cinéma a produit en utilisant des paysages à la fois extrêmement définis et relativement abstraits, symboles d'une géographie facilement identifiable par les spectateurs et cependant éloignée de leur expérience quotidienne.

Dépendamment de son traitement, la représentation urbaine isole ou assimile les personnages: elle est un arrière-plan idéal pour qui veut faire saisir rapidement le degré d'intégration sociale auquel s'est arrêté un individu. Il n'est pas surprenant qu'un cinéma de plus en plus attentif aux rapports des êtres humains à leur milieu ait largement privilégié la ville par rapport à la campagne. Moins facile à lire directement, plus varié, le 
monde rural est davantage physique que l'univers urbain : il est un climat, un ciel, une couleur, il porte les personnages au lieu de se poser en face d'eux. Ce que Calvino avait un peu confusément exprimé (on manque de recul quand on parle de ce au milieu de quoi on est plongé) était bien moins un retour au sens de la nature qu'une correspondance, évidemment fictionnelle, entre des individus et leur entourage. Sans faire nécessairement de la psychologie, les films qui réservent une large place à l'environnement problématisent leurs personnages. Au lieu de reprendre toutes les œuvres déjà citées, on se contentera d'évoquer brièvement l'exemple d'Antonioni. Nombre de ses films évoquent une perte: quelque chose, qui n'est pas toujours concrètement désigné, a été égaré et ne se retrouvera pas. Le Amiche est un film de ville où l'ensemble des jeunes femmes se définissent en fonction de l'ambiance turinoise. Mais la seule d'entre elles qui ne s'adapte pas, qui s'égare et ne peut se retrouver, est condamnée par ses compagnes lors d'une excursion à la mer où la sécheresse de la plage et la maigreur d'une végétation mal venue dans le sable ôtent aux participants leur apparence urbaine (aux deux sens de l'adjectif), tout en dénonçant ce qui ne sera pas récupéré. Les deux protagonistes de Il Grido et du Deserto rosso conduisent une recherche vaine dans une Padana sans horizon. L'infinitude est ici fondamentale: la plaine ne saurait avoir la variété dont font état les historiens puisqu'elle est l'emblème du rien. L'Avventura enfin met en œuvre une séparation encore plus marquée, une absence inexplicable dans un Sud (les îles éoliennes) qu'on traverse sans réussir à le voir. L'essentiel, dans ces quatre films, est la distance infranchissable inscrite dans le paysage: l'entourage ne correspond pas à l'état psychologique des personnages, il est l'image, la forme même de leur éloignement.

Le paysage est constitué d'éléments naturels: sol, plantes, arbres. Et si les hommes ont depuis des millénaires travaillé à en modifier l'aspect, à le domestiquer, ils ont pourtant tardé à l'envahir. Comme le souligne Deserto rosso, l'usine a substitué un décor au paysage. L'Italie du miracle puis celle de la crise, au même titre que les autres pays industrialisés, a concentré son activité dans des lieux artificiellement aménagés, lieux-objets peuplés d'objets qui sont ceux où habite désormais la majorité 
de la population. La transition s'est faite, à vive allure, dans le troisième quart $\mathrm{du} \mathrm{XX}^{\mathrm{e}}$ siècle, cette époque où quelques films, parmi ceux dont le grand public se souciait peu, ont mis en jeu la fonction symbolique de l'environnement. Traiter les paysages en images pour les intégrer au récit revenait à naviguer à contrecourant de la mode, du développement touristique et même de la réflexion historique. Mais un tel choix demeurait alors compréhensible; il faisait signe à ceux qui voulaient bien s'y intéresser. Depuis l'avènement sans partage de la ville-objet, ce symbolisme est probablement devenu illisible, le paysage arrière-plan est acceptable, le paysage rôle ne l'est pas. Ou bien, s'il intervient parfois, c'est dans un film historique, comme rappel d'une nature qui a cessé d'être le milieu où nous vivons.

Université de Paris III

\section{RÉFÉRENCES BIBLIOGRAPHIQUES}

Biasutti, 1962 : Renato Biasutti, Il Paesaggio terrestre, Torino, UTET, 1962.

Brunetta, 1972: Gian Piero Brunetta, Intellettuali, cinema e propaganda tra le due guerre, Bologna, Patron, 1972.

Brunetta, 1980 : Gian Piero Brunetta, "La emigrazione dei generi dalla biblioteca alla filmoteca dell'Italiano", Italian Quarterly, vol. XXI, n 81, p. 83-90.

Brunetta, 1993: Gian Piero Brunetta, Storia del cinema italiano II. Il cinema del regime, 1929-1943, Roma, Editori Riuniti, 1993.

Calvino, 1980 : Italo Calvino, Una pietra sopra, Torino, Einaudi, 1980.

Gambi et Ginzburg, 1979: Lucio Gambi et Carlo Ginzburg, LItalia, regioni e paesaggi, Bologna, Zanichelli, 1979.

Pavese, 1952: Cesare Pavese, Il Mestiere di vivere, Torino, Einaudi, 1952.

Pugh, 1988: Stephen Pugh, Reading Landscape, Country-City-Capital, Manchester University Press, 1988.

Quasimodo, 1971 : Salvatore Quasimodo, Poesie. Discorsi sulla poesia, Milano, Mondadori, 1971.

Ritter, 1963 : Joachim Ritter, "Zur Funktion des Ästhetischen in der modern Gesellschaft", Schriften der Gesellschaft zur Förderung der Westfälischen Landesuniversität zu Münster, Münster, 1963, p. 24-27.

Scimone, 1964: Giuseppe Scimone, "The Italian Miracle", dans Josselyn Hennessy, Vera Lutz et Giuseppe Scimone (dir.), Economic "Miracles", London, The Institute of Economic Affairs, 1964.

Sereni, 1965 : Emilio Sereni, Storia del paesaggio agrario italiano, Paris, Julliard, [1960] 1965.

Wall, 1997 : Anthony Wall, "Dear Antonioni ", BBC 2, série Arena, Émission du 27 novembre. 